Journal of Behavioral Data Science, 2021, 1 (2), 31-53.

DOI: https://doi.org/10.35566/jbds/v1n2/p3

\title{
Tree-based Matching on Structural Equation Model Parameters
}

\author{
Sarfaraz Serang ${ }^{1[0000-0002-7985-4951]}$ and James Sears ${ }^{2[0000-0002-0087-1354]}$ \\ 1 Utah State University, Logan, UT 84322, USA \\ sarfaraz.serang@usu.edu \\ 2 University of California, Berkeley, CA 94720, USA \\ james.sears@berkeley.edu
}

\begin{abstract}
Understanding causal effects of a treatment is often of interest in the social sciences. When treatments cannot be randomly assigned, researchers must ensure that treated and untreated participants are balanced on covariates before estimating treatment effects. Conventional practices are useful in matching such that treated and untreated participants have similar average values on their covariates. However, situations arise in which a researcher may instead want to match on model parameters. We propose an algorithm, Causal Mplus Trees, which uses decision trees to match on structural equation model parameters and estimates conditional average treatment effects in each node. We provide a proof of concept using two small simulation studies and demonstrate its application using COVID-19 data.
\end{abstract}

Keywords: Matching - Structural Equation Modeling · Decision Trees · Machine Learning

\section{Introduction}

Understanding the causal effect of a treatment has historically been of great scientific interest and remains one of the most frequently pursued objectives in scientific research today. The gold standard for evaluating treatment effects is the randomized controlled trial, where the researcher randomly assigns treatment status to each individual. The benefit of this approach is that the causal effect of the treatment can be estimated by simply comparing outcomes between those who were treated and those who were not (Greenland, Pearl, \& Robins, 1999). Random assignment of treatment guarantees that, on average, the treated and untreated individuals will be equal on all potential confounding variables, both measured and unmeasured. Eliminating the possibility of confounding clears the way for a direct comparison to be made.

However, random assignment is not always possible. This can be for ethical reasons, since researchers cannot, for example, force participants to smoke to 
investigate the effects of smoking. It can also be for practical reasons, where the researcher cannot control the assignment of a treatment. For example, researchers cannot randomly assign depression to some participants, enact a law or policy in a randomly assigned jurisdiction, or choose where their participants live. An observational study, where treatment is not randomly assigned, may be the only available option in these cases. Unlike randomized controlled trials, direct comparisons between treated and untreated individuals in an observational study cannot be made as easily. This is because treated and untreated participants may not be equal in all other characteristics, creating the potential for confounding effects. In fact, it may be differences in these very characteristics that lead some participants to select treatment, making the estimation of the treatment's effect less straightforward. To estimate a treatment's effect, it must first be defined, which we do in the context of the potential outcomes framework.

\subsection{Potential Outcomes Framework and Assumptions}

The foundations for the potential outcomes framework were laid out by Neyman, Iwaszkiewicz, and Kolodziejczyk (1935) and further developed by Rubin (1974), resulting in it also being called the Rubin Causal Model, Neyman-Rubin Causal Model, and Neyman-Rubin counterfactual framework of causality. The model can be conceptualized as follows. Let $Y_{1 i}$ be the potential outcome of individual $i$ if they received the treatment and $Y_{0 i}$ be the potential outcome of individual $i$ if they did not receive the treatment. The observed score $Y_{i}$, can be written as

$$
Y_{i}=W_{i} Y_{1 i}+\left(1-W_{i}\right) Y_{0 i}
$$

where $W_{i}=1$ if the individual received treatment and $W_{i}=0$ if they did not. $W_{i}$ simply acts as an indicator variable denoting the receipt of treatment. The term treatment here and throughout the paper is used rather loosely and can be used interchangeably with exposure.

The effect of the treatment is simply $Y_{1 i}-Y_{0 i}$, the difference between the potential outcomes if the individual had received treatment and if they had not. The fundamental problem of causal inference, as stated by Holland (1986), is that it is impossible to observe both $Y_{1 i}$ and $Y_{0 i}$ for the same individual. If the individual received treatment, we can observe $Y_{1 i}$, but not its counterfactual, $Y_{0 i}$. The inverse is also true: if the individual did not receive treatment, we can observe $Y_{0 i}$, but not its counterfactual, $Y_{1 i}$. Therefore, it is impossible to observe the effect of the treatment on the individual. As an example, we can see that it is impossible to observe the effect of divorce on a child's academic test scores because at a given moment in time, the parents can either be divorced or not divorced, but not both. We cannot observe the test scores under both conditions, so we cannot observe the effect of divorce on that child's scores.

Though we cannot observe the effect of the treatment on a given individual, we can estimate the average treatment effect (ATE) on a population. The ATE is the average effect expected from taking a population where no individuals received the treatment and providing the treatment to all of them (Austin, 2011). 
The ATE is defined as $A T E=E\left(Y_{1 i}-Y_{0 i}\right)=E\left(Y_{1 i}\right)-E\left(Y_{0 i}\right)$, where $E(\cdot)$ is the expected value operator. Conceptually, this implies that although we cannot observe the treatment effect at the individual level, we can do so at a population level by using the average of the untreated participants as a proxy for the unobservable counterfactual (Guo \& Fraser, 2010). A related effect of interest in this paper is the conditional average treatment effect, or CATE, (Abrevaya, Hsu, \& Lieli, 2015), defined as $C A T E=E\left(Y_{1 i}-Y_{0 i} \mid \boldsymbol{X}_{i}\right)$, where $\boldsymbol{X}_{i}$ is a vector of covariates. The CATE allows us to evaluate heterogeneity in treatment effects between subpopulations, for example, allowing for separate estimation of the ATE in males and females if they are believed to be different.

One important assumption of the potential outcomes framework is the Stable Unit Treatment Value Assumption, or SUTVA (Rubin, 1980, 1986). It represents the assumption that the potential outcomes would be the same no matter how an individual came to be assigned to a treatment, and no matter what treatments are received by other individuals. It assumes that neither treatment assignment mechanisms nor social interactions affect potential outcomes. Another assumption, one we give more attention due to the focus of this paper, is known as the strong ignorability assumption (Rosenbaum \& Rubin, 1983). Treatment assignment is strongly ignorable if two conditions collectively hold. The first condition is $\left(Y_{0}, Y_{1}\right) \perp W \mid \boldsymbol{X}$, that treatment assignment is independent of the potential outcomes conditional on covariates. The second condition is $0<P(W=1 \mid \boldsymbol{X})<1$, that every participant has a nonzero probability of receiving either treatment, conditional on covariates.

The necessity of the conditional independence piece of the strong ignorability assumption becomes evident when considering the necessary conditions for using untreated participants as a proxy for the counterfactual. To estimate the ATE by taking the difference between the averages of treated and untreated participants, we implicitly assume that the average scores produced by the untreated participants are an unbiased estimate of what the average scores produced by the treated participants would have been had they not received the treatment. In doing so, we must ensure that the treated and untreated participants are similar in relevant characteristics, so that the untreated participants can serve as a faithful representation for their treated counterparts. For example, if the treated group contained only males and the untreated group contained only females, using the untreated group as a proxy for the treated group might not produce a fair comparison, depending on what is being studied. This is why randomized controlled trials are considered the gold standard: random assignment ensures that, on average, all such possible confounders are balanced, making the treated and untreated participants comparable.

As pointed out by Thoemmes and Kim (2011), the strong ignorability assumption cannot be empirically tested. This is because treatment assignment must be conditionally independent of all relevant covariates both observed and unobserved, and it is not possible to empirically verify that variables that are not collected do not play a role. As such, researchers who attempt to justify this assumption are limited to making a convincing argument that they have mea- 
sured the relevant covariates and showing that these are balanced across treated and untreated participants. The most common way of demonstrating balance in an observed covariate across groups is via a standardized mean difference. This takes the form of the mean difference in the covariate between groups (in absolute value) divided by either a pooled standard deviation or an unpooled standard deviation of one of the groups.

A standardized mean difference of 0 would indicate the covariate has the same mean across groups. However, there is no universally agreed upon metric for judging how small a nonzero standardized mean difference must be to be considered negligible enough for the groups to be considered balanced on the covariate for practical purposes. Many recommendations exist in the methodological literature. Harder, Stuart, and Anthony (2010) use a value less than 0.25, based on a suggestion by Ho, Imai, King, and Stuart (2007). Austin (2011) suggests a stricter value of less than 0.1, based on work by Normand et al. (2001). Leite, Stapleton, and Bettini (2018) point out that for educational research, the What Works Clearinghouse Procedures and Standards Handbook (version 4.0) requires a value less than 0.05 without additional covariate adjustment, or between 0.05 and 0.25 with additional regression adjustment (U.S. Department of Education, Institute of Education Sciences, \& What Works Clearinghouse, 2017).

Analyzing standardized mean differences is reasonable when attempting to balance across demographic covariates such as sex, age, race, etc. Yet some characteristics do not lend themselves well to being assessed in this way. Consider an example where we are interested in evaluating the effects of a breakup from a romantic relationship (the treatment) on life satisfaction (the outcome). For simplicity, let us assume that we only collect data from one partner per couple. Putting demographics aside, affect might be an important covariate to balance on. However, ensuring that couples who do and do not break up have the same average affect might not be especially useful. Stability of affect has been shown to be predictive of whether couples remain together or break up (Ferrer, 2016; Ferrer, Steele, \& Hsieh, 2012). That is, fluctuations in affect are what need to be balanced, not simply average affect. Consider the plot given in Figure 1 of two hypothetical individuals, $\mathrm{J}$ and $\mathrm{K}$, and their affect over time. J has highly variable affect, whereas $\mathrm{K}$ has relatively stable affect. Based on the aforementioned research, $\mathrm{J}$ is more likely to experience a breakup, given their instability. However, both $\mathrm{J}$ and $\mathrm{K}$ have the same average affect. Imagine a treatment group filled with individuals like $\mathrm{J}$ and an untreated group filled with individuals like $\mathrm{K}$. According to the standardized mean difference, these two groups would be balanced across affect, because they have the same mean affect. The fact that they have different patterns with regard to the variability would be entirely missed.

The literature does recommend that covariates should be balanced across groups on not just the mean, but the distribution of the variables (Austin, 2011; Ho et al., 2007). Researchers are encouraged to examine higher-order moments, as well as interactions between covariates. Graphical methods are often used 


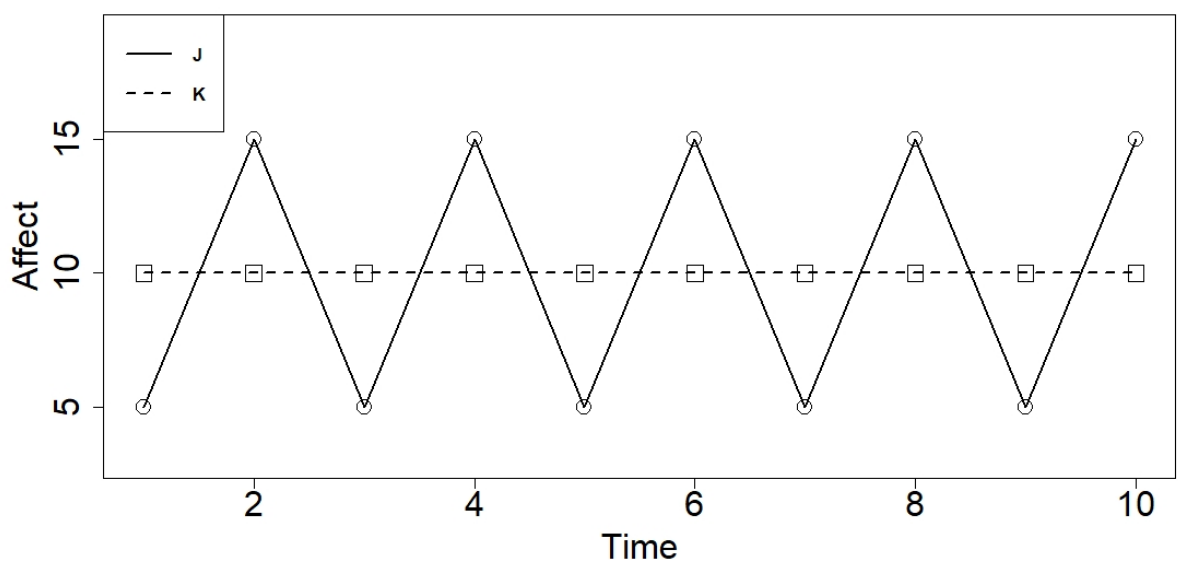

Figure 1. Stability of Affect in Two Hypothetical Individuals

to make these comparisons, including quantile-quantile plots, boxplots, density plots, etc. Though visualizations can be helpful for univariate or even bivariate data, they become less useful with higher-dimensional data, as in our example. Furthermore, in this case, they do not quite address the issue directly. We would like to balance on stability of affect, which is not entirely captured by either univariate higher order moments or interactions.

\subsection{Purpose}

Although conventional approaches can be useful when balancing on demographic variables and other such covariates, they are not as well suited for balancing on more complex functions of the data, such as stability of affect. This paper seeks to develop an approach that allows us to balance on more flexibly defined characteristics of interest. We begin by reviewing some classic and recent approaches to matching. We then provide an introduction to structural equation model trees and their variations. Drawing from these, we propose our own algorithm, Causal Mplus Trees, and describe its implementation. We then conduct two small simulation studies demonstrating our algorithm's effectiveness and an empirical analysis of COVID-19 data. We conclude with a discussion of practical recommendations and future directions.

\subsection{Propensity Score Matching}

Thus far we have discussed ways to evaluate whether treated and untreated participants are balanced on covariates. If they are found to be unbalanced, we can turn to statistical approaches to balance them. A natural initial thought 
would be to use ordinary least squares regression, conditioning on covariates within the model. However, Berk (2004) points out that simply calculating a conditional distribution of the outcome is not sufficient to draw causal inference and that stronger assumptions are needed.

A popular alternative is to use propensity scores, defined as the probability of treatment conditional on observed covariates (Rosenbaum \& Rubin, 1983). It has been shown that propensity scores can balance treated and untreated participants in the sample, and that both treatment assignment as well as observed covariates are conditionally independent given the propensity score (Rosenbaum \& Rubin, 1983). This implies that for participants with the same propensity score, the mean difference in the outcome between treated and untreated participants is an unbiased estimate of the ATE at that propensity score (Guo \& Fraser, 2010). Propensity scores are typically calculated using logistic regression, with the observed covariates predicting treatment status $(W)$. The estimated regression coefficients are then used as weights in a model predicting the probability of treatment for each individual. The estimated propensity score is this predicted probability of treatment.

Once propensity scores have been calculated, they can be used in various ways, including propensity score matching (Rosenbaum \& Rubin, 1985), stratification on the propensity score (Rosenbaum \& Rubin, 1984), and inverse probability of treatment weighting using the propensity score (Hirano \& Imbens, 2001). Of these three, propensity score matching seems to eliminate more of the systematic differences in covariates (Austin, 2009) and also seems to be the most popular (Thoemmes \& Kim, 2011), so we limit our focus to propensity score matching. Propensity score matching involves finding treated and untreated participants with similar propensity scores to use as each other's counterfactuals. According to Austin (2011) and the systematic review conducted by Thoemmes and Kim (2011), the most commonly used form of matching is 1:1 matching, where each treated participant is matched with a single untreated participant, forming a pair. Thoemmes and Kim (2011) found that the most popular way to do this in the social sciences was to use greedy matching, in which a treated subject is selected at random and the untreated subject with the closest propensity score is paired with them. The process is repeated until all treated subjects have a match. This is in contrast to optimal matching, where matches are selected to optimize the distance between propensity scores for the entire sample, which has been shown to perform comparably to greedy matching (Gu \& Rosenbaum, 1993). The 1:1 matching scheme produces pairs of treated and untreated participants who should in theory be balanced on the propensity scores. The ATE can then be estimated simply by performing a paired $t$ test (Austin, 2011).

Of course, one must still ensure that the propensity scores are balanced across treated and untreated participants. If they are not, it is recommended that the logistic regression model be iteratively refined by including nonlinear terms and interactions between covariates until balance has been achieved (Austin, 2011; Rosenbaum \& Rubin, 1984, 1985; West et al., 2014). Latent variable models can be used to calculate propensity scores by balancing on latent covariates whose 
scores are estimated via factor score estimation (Raykov, 2012), or by using structural equation modeling to estimate propensity scores directly (Leite et al., 2018). Machine learning techniques including bagging, boosting, trees, and random forests, have also been used for the estimation of propensity scores (Lee, Lessler, \& Stuart, 2010).

\subsection{Causal Trees}

A recent alternative to propensity score matching is the causal tree approach proposed by Athey and Imbens (2016). Essentially, they use decision trees to partition the sample into groups of individuals who are similar on important dimensions. They then treat these groupings as matched, and use them to estimate the ATE. Decision trees (Breiman, Friedman, Olshen, \& Stone, 1984), use recursive partitioning to separate a predictor space into regions that are as homogeneous as possible on a target variable of interest. Binary splits are made on predictors (e.g. female vs. male, age $\leq 60$ vs. age $>60$, etc.), splitting the sample into two nodes. All possible splits are made on all predictors, and the split that makes the resulting samples in each node as homogeneous as possible is presented as a candidate split. If this split exceeds a predetermined fit criterion, the split is made, partitioning the sample into the two daughter nodes. Otherwise, the split is not made, and the parent node becomes a terminal node. The process continues recursively on each daughter node until all nodes are terminal nodes. We refer readers to Serang et al. (2021) for additional description of the procedure.

Decision trees are most often used for prediction of a target variable. The critical insight of Athey and Imbens (2016) is that trees have a natural proclivity for creating homogeneous subgroups. Instead of trying to predict a target variable, we can substitute the vector of covariates, $\boldsymbol{X}$. The tree will then produce terminal nodes where the observations in each terminal node are as similar as possible on the covariates, achieving the same aim as matching. Each terminal node is characterized by splits on predictors (separate from $\boldsymbol{X}$ ) that define membership in that node. In what they call an honest approach to estimation, the authors recommend that these subgroup definitions be applied to a fresh holdout sample not involved in the construction of the tree, to create subgroups using the new data. CATEs (ATEs conditional on subgroup membership) can then be estimated in each subgroup via mean differences between treated and untreated participants within the subgroup. Causal inference can also be drawn using standard approaches, such as an independent-samples $t$ test.

The advantage of causal trees over propensity score methods is that one need not worry about the estimation of or balancing on propensity scores. Propensity scores only serve as a middleman in propensity score matching, and causal trees use the properties of decision trees to bypass them entirely. Additionally, causal trees easily accommodate heterogeneity in causal effects. In our running example, we wish to match on stability of affect. If we use demographic variables as splitting variables in the tree, we can potentially find subgroups defined by these demographic characteristics (e.g. sex, age, etc.) that have different levels of affect 
stability. The causal tree approach would then allow us to estimate the causal effect of a breakup separately in each of these subgroups, as well as compare them to see if the causal effect differs by subgroup.

\subsection{Structural Equation Model Trees}

One limitation of causal trees as described is that they assume we wish to match on observed covariates. However, stability in our example is not an observed variable in the data: it is a characterization based on a pattern. One way to characterize stability for the data in our example would be to fit a simple intercept-only growth curve model and examine the residual variance. A model fit to individuals such as $\mathrm{J}$ would produce a large residual variance, whereas a model fit to individuals like $\mathrm{K}$ would yield a relatively small residual variance. Thus, stability of a group can be characterized by model-based parameter estimates, in lieu of observed variables.

To do this within the causal tree framework, we would need a mechanism to fit a model within each node. For longitudinal models, we can use an approach like the nonlinear longitudinal recursive partitioning algorithm proposed by Stegmann, Jacobucci, Serang, and Grimm (2018), which allows the user to fit linear and nonlinear longitudinal models within each node. A more general approach is the structural equation model tree (SEM Tree) proposed by Brandmaier, Oertzen, McArdle, and Lindenberger (2013), which allows for structural equation models (SEMs) to be fit within each node. A benefit of the latter is the flexibility of the SEM framework, which can accommodate a wide range of models, including many longitudinal models, via latent growth curve modeling (Meredith \& Tisak, 1990).

The logic of SEM Trees is similar to that of standard decision trees, with some minor variations. A prespecified SEM is first fit to the full sample, and the minus two $\log$-likelihood $(-2 \log L)$ is calculated. Then, the $-2 \log L$ for the candidate split is calculated. Since the split can be conceptualized as a multiple group model (Jöreskog, 1971), the $-2 \log L$ for the split is simply the sum of the $-2 \log L$ values for each daughter node. A likelihood ratio test is then conducted with these two $-2 \log L$ values. If it rejects, the split is made. As in other decision trees, this process is recursively repeated until all daughter nodes are terminal nodes. Unlike conventional decision trees, terminal nodes in SEM Trees do not provide a predicted proportion or mean. Rather, each terminal node is characterized by a set of parameter estimates for the SEM fit to the sample in that node. In this way, SEM Trees can be used to identify subgroups of people who are similar in that they can be represented by a set of parameter estimates that is distinct from the parameter estimates that characterize those in

other nodes. SEM Trees can therefore identify subgroups with distinct patterns of stability, growth, or other patterns reflected in the parameter estimates. 


\subsection{Mplus Trees}

The SEM Trees algorithm is implemented in the semtree (Brandmaier, Prindle, \& Arnold, 2021) package in R (R Core Team, 2020). The SEMs are fit in either the OpenMx package (Neale et al., 2016) or the lavaan package (Rosseel, 2012). The OpenMx package is flexible but challenging to use, especially for casual users, given the need to specify the entirety of the model with limited defaults. The lavaan package is much easier to use given the ease with which one can specify models, however it is currently more limited in the scope of the models it can fit. The MplusTrees package (Serang et al., 2021) is an implementation of SEM Trees which uses Mplus (Muthén \& Muthén, 1998-2017) to fit the models, the rpart package (Therneau \& Atkinson, 2018) to perform the recursive partitioning needed to grow the trees, and the MplusAutomation package (Hallquist \& Wiley, 2018) to interface between R and Mplus. MplusTrees capitalizes on the wide variety of complex models that can be specified in Mplus, the ease with which they can be specified, and the currently superior estimation algorithms it uses for fitting these models.

The Mplus Trees algorithm itself (Serang et al., 2021) is very similar to the SEM Trees algorithm (Brandmaier et al., 2013). However, one key difference is the criterion used for splitting. Although the MplusTrees package also has the capability to split using the likelihood ratio test, this is not the primary method. Instead, Mplus Trees uses a complexity parameter, $c p$. This $c p$ parameter is a proportion specified in advance by the user. A split will be made if that split improves on the -2LogL of the full sample (the parent node) by at least $c p$ times that $-2 \log L$. Smaller values of $c p$ result in more splits since a relatively smaller improvement in the $-2 \log L$ is needed for a split to be made, whereas larger values lead to fewer splits. As such, the use of $c p$ serves more as a heuristic than a formal test based on statistical significance. Ideally, $c p$ would be selected by cross-validation, and this functionality is available in the MplusTrees package. However, long computational times may require users to simply try a handful of $c p$ values and select the most appropriate one given the context.

\section{Causal Mplus Trees}

We now propose our own matching algorithm, Causal Mplus Trees, using Mplus Trees to create causal trees that match on parameters from an SEM, and estimating CATEs in a holdout sample. We begin by first randomly partitioning the dataset into two parts, one subsample to perform the matching and the other to perform the estimation of the CATEs. In most cases, the matching subsample will require more participants, since fitting an SEM and building a decision tree is more sample intensive than estimating a mean difference. We suggest devoting $80 \%$ of the sample to the matching subsample and $20 \%$ to the estimation subsample, though this ratio can be adjusted depending on the complexity of the SEM, the overall sample size, etc.

Beginning with the matching subsample, we can partition $\boldsymbol{X}$ into two parts: $\boldsymbol{X}_{M}$, the modeled covariates modeled in the SEM whose parameters we wish to 
match on, and $\boldsymbol{X}_{S}$, the splitting covariates we want to split on in the recursive partitioning process which define the subgroups of the tree's terminal nodes. Guidance for whether a covariate should be a modeled covariate or a splitting covariate is provided in the discussion. Let $M$ be an SEM with parameters $\boldsymbol{\theta}$ that produces $\boldsymbol{X}_{M}$, so that $M(\boldsymbol{\theta})=\boldsymbol{X}_{M}$. In our running example, $M$ would be the intercept-only growth model and $\boldsymbol{\theta}$ would be its parameters. For properly specified $M, \boldsymbol{X}_{M}$ can be used to estimate $\boldsymbol{\theta}$, resulting in parameter estimates $\hat{\boldsymbol{\theta}}$. Using Mplus Trees, we can build a tree that matches on $\hat{\boldsymbol{\theta}}$, with groups (terminal nodes) defined by their covariate patterns on $\boldsymbol{X}_{S}$. The treatment assignment information, $W$, is not provided to the recursive partitioning algorithm and so the tree is built blind to $W$. In the estimation subsample, we can divide participants into groups according to the splits found by the tree. Within each group, we can estimate the CATE as defined before by taking the difference between the means of the outcomes of the treated and untreated participants in each group. Since we are using a fresh sample, we can draw inference using hypothesis tests such as an independent-samples $t$ test or another suitable alternative. We can also test whether the CATE differs by group by testing the interaction effect in a two-way independent ANOVA.

\section{Simulation Studies}

As a proof of concept for Causal Mplus Trees, we performed two small simulation studies. The simulation studies were conducted in $\mathrm{R}$ using the lavaan package to simulate data and the MplusTrees package for analysis. Readers are referred to the package documentation for details regarding the implementation of the algorithm in the software. Each simulation consisted of 1,000 replications.

\subsection{Longitudinal Simulation}

The first simulation mapped onto our running example regarding stability of affect. Each sample consisted of $N=2,000$ individuals, 1,000 in each of two groups. The data were generated from an intercept-only (no growth) model with 10 time points. The intercept had a mean of 10 with a variance of 1 . The only difference between the groups was in the residual variance, $\sigma_{\epsilon}^{2}$. One group had a residual variance of 1 (the group with stable affect), and the other had a residual variance of 10 (the group with unstable affect). The group memberships were identified by a dichotomous covariate, used as a splitting variable. Thus, the tree matched on the growth curve, using the group membership to split. Within each group, treated and untreated participants were evenly split (500 each). A diagram of this population tree is given in Figure 2. For the stable affect group, outcomes were generated using a standard normal distribution, $N(0,1)$, for the untreated group and a $N(0.5,1)$ distribution for the treated group, to represent a medium-sized CATE. However, for the unstable affect group, the outcome distributions were flipped, with the untreated group's outcome being generated from a $N(0.5,1)$ distribution, whereas the treated group's outcome 
was generated from a $N(0,1)$ distribution. In this way, although the ATE for the full sample was 0 , the CATE for each group was 0.5 in absolute value.

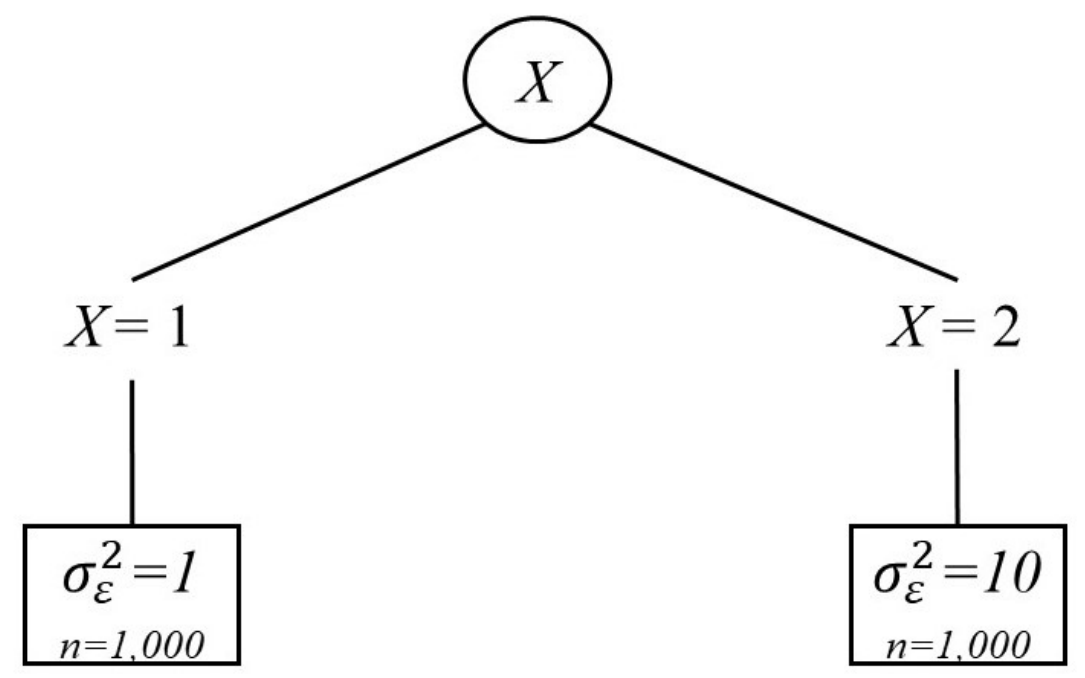

Figure 2. Population Tree for Longitudinal Simulation

It should be noted that these groups are, from the start, balanced on the modeled covariates. Since the growth curve variables were all generated to have a mean of 10, they would be considered balanced according to the standardized mean difference. Thus, if one were to follow conventional procedure, propensity scores would not be needed here, and the estimation of the ATE would consist of simply the mean difference between treated and untreated participants, which would be 0 on average.

The Causal Mplus Trees algorithm was implemented as described in the prior section, with $80 \%$ of the sample (1,600 individuals) used for matching and $20 \%$ (400 individuals) used to estimate CATEs. A $c p$ value of .01 was used to split, with a minimum of 100 individuals required to consider splitting on a node. Each terminal node was also required to have at least 100 individuals within it. For each replication, the CATE was estimated in each group using an independent samples $t$ test. A two-way independent ANOVA was also conducted to determine if CATEs differed by group.

Overall, the results demonstrated the effectiveness of the algorithm. Across all replications, $94.5 \%$ of CATEs were detected. Additionally, $99.8 \%$ of the interactions from the two-way ANOVA were detected, showing that the algorithm can detect differences in CATEs by group. As a comparison, we also analyzed 
these data as they would have been analyzed using the conventional approach. Since the covariates were on average balanced according the standardized mean difference, the ATE would have been estimated by using the full sample to estimate the mean difference between treated and untreated participants. Despite a sample size of 2,000 to do this (relative to the only 400 available to Causal Mplus Trees after performing the matching), only $3.4 \%$ of datasets yielded statistically significant ATEs, consistent with a nominal false positive rate of $5 \%$.

\subsection{Measurement Simulation}

The second simulation study is similar to the first, but used a measurement model as opposed to a longitudinal model for the matching. For the second study, each sample consisted of $N=3,000$ individuals, divided into three groups. One group (the small loading group) contained 1,500 individuals, while the remaining two groups (the medium and large loading group) each contained 750. Data were generated from a one-factor confirmatory factor analysis model with 15 items. Factor variances were fixed to 1 , and uniquenesses were also simulated to be 1. As implied above, the only differences were in the loadings, $\lambda$. In the small loading group, all loadings were simulated to be 0.1 , in the medium loading group they were 0.5 , and in the large loading group they were 0.9 . The model was generated to reflect the case where items are more related to a latent construct for some people than for others. If the latent variable were a psychological disorder, this would map onto the idea that the items better reflect the presence of that disorder in some groups relative to others.

As with the previous simulation study, a single splitting covariate denoting group membership was used as the splitting variable, albeit with three values given the three groups. Figure 3 shows a diagram for this population tree. As with the other simulation study, each group was evenly divided on treated and untreated participants. In the small loading group untreated participants had outcomes generated from a $N(0,1)$ distribution, whereas the treated group's outcome was generated from a $N(0.5,1)$ distribution. In the medium and large loading groups this was reversed: untreated participants had outcomes from a $N(0.5,1)$ distribution whereas treated participants had outcomes from a $N(0,1)$ distribution. In this way, these samples too had an average ATE of 0, in addition to being on average balanced on the modeled covariates according to the standardized mean difference, since all items had an average score of 0 .

The algorithm again used $80 \%$ of each sample (2,400 participants) for matching and 20\% (600 participants) for estimation. As before, a minimum of 100 individuals was required to consider splitting a node and in each terminal node, however this study used a $c p$ value of .001 . Unlike the previous study where the split was made in every replication, the algorithm had some slight trouble finding all the groups in this study. All three groups were found in $92.7 \%$ of simulations, but only two groups were found in the remaining $7.3 \%$. Among all the groups found, $88.3 \%$ of the CATEs were detected, along with $99.7 \%$ of the interactions. Alternatively, when using the entire sample to calculate the ATE, only $3.5 \%$ of simulations yielded significant results. These results are similar 
to those found in the first simulation study. Taken together, they show that the Causal Mplus Trees algorithm is able to estimate CATEs and support hypothesis testing to determine their statistical significance. It can also determine whether the CATEs differ by group. Notably, CATEs were found in the absence of ATEs, with modeled covariates already balanced across treated and untreated participants according to the standardized mean difference.

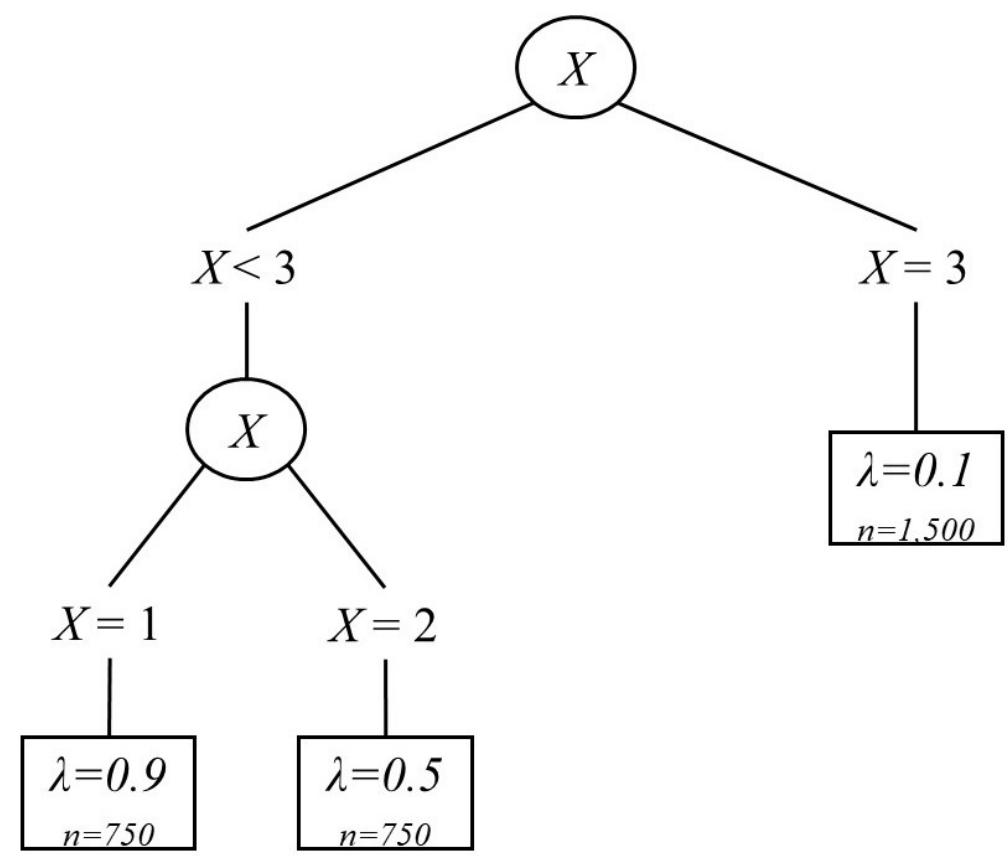

Figure 3. Population Tree for Measurement Simulation

\section{Empirical Example}

As an illustration of how Causal Mplus Trees can be used in practice, we present an analysis of COVID-19 data. The dataset contains information from four different sources: public health data from the COVID-19 Data Repository by the Center for Systems Science and Engineering (CSSE) at Johns Hopkins University (Dong, Du, \& Gardner, 2020), demographic data from the 2010 US Decennial Census (U.S. Census Bureau, 2010), governor's party information obtained from the National Governors Association Roster (National Governors Association, 2020), and mobility data from Unacast, a location data analytics company (Unacast, 2020). 
To capture how individuals' travel activity patterns responded to the spread of COVID-19, we utilized Unacast's measure of the change in average distance traveled. Travel distance was measured using the GPS positions of millions of mobile devices and aggregated each day to a county-level average. For a detailed overview of variable construction and discussion of potential sources of bias, see Sears, Villas-Boas, Villas-Boas, and Villas-Boas (2020). The data were analyzed at the county level, consisting of 3,030 counties or county-equivalents from all 50 US states except Alaska. This represents over 95\% of counties in the US.

The goal of this analysis was to estimate the CATE of the governor's party (Democrat or Republican) on mobility in counties matched on the trajectory of COVID-19 cases early in the pandemic. We sought to answer the question: "for counties with similar trajectories of the rise in COVID-19 cases from March through June 2020, could differences in mobility in July 2020 be attributed to the governor's party?" Prior studies reveal strong links between political partisanship and the adoption of stay-at-home and social distancing orders as well as changes in residents' travel behavior and time spent at home (Adolph, Amano, Bang-Jensen, Fullman, \& Wilkerson, 2020; Allcott et al., 2020; Brzezinski, Deiana, Kecht, \& Van Dijcke, 2020; Gadarian, Goodman, \& Pepinsky, 2020). We provide a complementary analysis allowing us to understand whether the effect of gubernatorial political alignment extended beyond stay-at-home adoption timing to continued behavioral changes among constituents. Our analysis also examined how this effect differed across counties depending on demographic characteristics.

Case trajectories were modeled using the cumulative cases in the county divided by the population per 10,000 residents, hereafter referred to as COVID rates. COVID rates were calculated weekly from March 9, 2020 (around when states began reporting their first cases) until June 29, 2020, resulting in 17 time points of data per county. The SEM fit within each node of the tree was the logistic growth model given by

$$
\operatorname{COVID} D_{i}=\frac{\beta_{1 i}}{1+e^{-(t-\gamma) \alpha}}+\epsilon_{i}
$$

where $C O V I D_{i}$ is the COVID rate for county $i, \beta_{1 i}$ is the county-specific COVID rate when the "curve has flattened" (the upper asymptote), $t$ is the number of weeks $(t=1,2, \ldots, 17), \gamma$ is the inflection point, $\alpha$ is the rate of change, and $\epsilon$ is the residual. The model was specified using Taylor-series approximation (Browne \& Toit, 1991; Grimm \& Ram, 2009) with equal residual variances across time, $\sigma_{\epsilon}^{2}$, to aid estimation.

We used six demographic splitting variables: population (the total population of the county), white (the percentage of non-Hispanic Whites), age65_older (the percentage of people ages 65 years and older), median_inc (the median household income), bachelors (the percentage of people with at least a bachelor's degree), and rural (the percentage of the population considered rural). To reduce the computational burden of the algorithm, we reassigned values from 1 to 4 to each of these splitting covariates depending on the quartile in which they fell relative to the other counties. 
In implementing the Causal Mplus Trees algorithm, we used 2,424 counties to match the data and the remaining 606 to estimate the CATEs. We required that a minimum sample size of 300 was required to both attempt a split and to remain in each terminal node. A $c p$ value of .01 was used to split. The tree grown from the training data is given in Figure 4, with corresponding parameter estimates provided in Table 1. Group 1 consisted of those in the bottom three quartiles $(<93.1 \%)$ on white, below the median $(<17.2 \%)$ on age65_older, and in the bottom three quartiles of median_inc $(<\$ 53,601)$. It contained $29 \%$ of the counties, and was characterized by the highest asymptote, 61.33 cases per 10,000. Group 2 was made up of those in the bottom three quartiles $(<93.1 \%)$ on white, below the median $(<17.2 \%)$ on age65_older, but in the top quartile of median_inc $(>\$ 53,601)$. It represented $15 \%$ of the counties, and was characterized by the second highest asymptote, 50.19 cases per 10,000. Group 3 contained those in the bottom three quartiles $(<93.1 \%)$ on white, but above the median $(>17.2 \%)$ on age65_older. This group had $31 \%$ of counties, with the second lowest asymptote, 35.88 cases per 10,000. Group 4 consisted of those in the top quartile ( $>93.1 \%)$ on white, with $26 \%$ of counties and the lowest asymptote at 19.04 cases per 10,000. Group 4 also happened to be the most rural and least populated, potentially explaining the low asymptote.

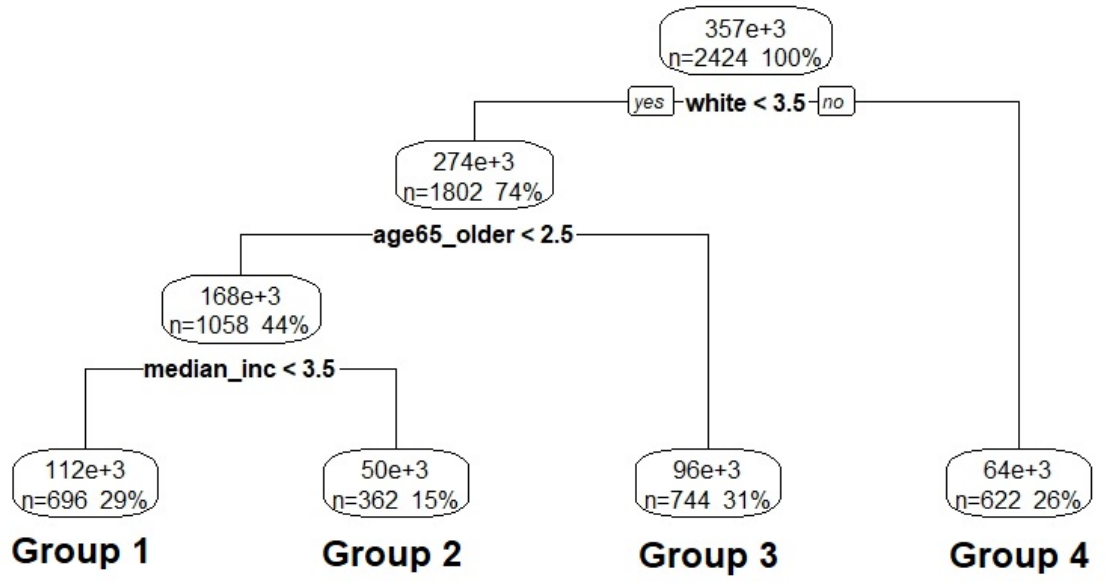

Figure 4. Tree from COVID-19 Data Matching Subsample

Governor's party (with Republican arbitrarily selected as the treatment) was used as the treatment variable in part because much of the policy, coordination, and messaging thus far has occurred via executive action at the state level. The outcome, mobility, was operationalized as the change in average distance traveled, or CADT. CADT for each day in July was calculated as the county- 
Table 1. Parameter Estimates for SEMs from the Groups in Figure 4

\begin{tabular}{ccccc}
\hline & Group 1 & Group 2 & Group 3 & Group 4 \\
\hline$n$ & 696 & 362 & 744 & 622 \\
$\beta_{1}$ (Mean) & 61.33 & 50.19 & 35.88 & 19.04 \\
$\beta_{1}$ (Variance) & $13,377.34$ & $7,767.81$ & $2,425.19$ & 455.58 \\
$\gamma$ & 9.33 & 6.94 & 10.23 & 10.44 \\
$\alpha$ & 0.69 & 0.56 & 0.44 & 0.40 \\
$\sigma_{\epsilon}^{2}$ & 554.05 & 139.86 & 88.02 & 18.73 \\
\hline
\end{tabular}

day level percentage point change in average travel distance relative to that day-of-week's average in early 2020 (average for Feb 10 to March 8, prior to the presence of COVID-19 in the US). Accordingly, a value of -3 indicates a 3 percentage point decline in average travel distance relative to baseline levels. A positive value of CADT signals that residents of that county increased their travel distances relative to their pre-COVID-19 patterns, whereas a negative value indicates reduced travel distances (that can occur through reductions in both the distances traveled per trip as well as the overall number of trips taken). Each county's average CADT for July was estimated by taking the mean of the daily CADT for each day from July 1, 2020 until July 31, 2020. The estimate of the CATE in each group, along with corresponding information, is given in Table 2 .

Table 2. CATEs and Significance Tests for COVID-19 Groups

\begin{tabular}{ccccc}
\hline & Group 1 & Group 2 & Group 3 & Group 4 \\
\hline$n_{\text {Rep }} ; n_{\text {Dem }}$ & $104 ; 59$ & $58 ; 55$ & $104 ; 94$ & $76 ; 60$ \\
$\overline{C A D T}_{\text {Rep }}$ & $-0.93 \%$ & $-4.47 \%$ & $-0.58 \%$ & $-0.78 \%$ \\
$\overline{C A D T}_{\text {Dem }}$ & $-2.47 \%$ & $-10.92 \%$ & $0.88 \%$ & $-2.30 \%$ \\
CATE & $1.54 \%$ & $6.46 \%$ & $-1.46 \%$ & $1.53 \%$ \\
$t$ test & $t(104.08)=0.94 t(108.76)=2.84$ & $t(186.87)=-0.86$ & $t(122.99)=1.15$ \\
$p$ value & .349 & .006 & .389 & .252 \\
\hline
\end{tabular}

Of the four groups, the only one with a statistically significant CATE was Group 2, where counties in states with Democratic governors had an average CADT that was 6.46 percentage points less than counties in states with Republican governors $t(108.76)=-2.84, p=.006$. Group 2 was on average the most populous, least rural group of the four, as well as the most educated with highest median incomes. As such, Group 2 contained the country's more metropolitan areas. We interpret this result to mean that in metropolitan counties matched for COVID rates, people in counties in states with Democratic governors traveled 6.5 percentage points less in July than people in comparable counties in states with Republican governors. Of note, the two-way independent ANOVA found that in the estimation subsample, a significant main effect of party was not found $F(1,598)=3.76, p=.053$, whereas a main effect of Group $F(3,598)$ 
$=13.45, p<.001$, and an interaction $F(3,598)=3.41, p=.017$ were. This suggests that the party effect is more prominent for more metropolitan counties, but would be obscured if examining the country as a whole. The mean difference between parties in CADT for all 3,030 counties was only 0.60 percentage points, with a $t$ test on the full dataset yielding $t(2422.6)=-1.50, p=.133$, though this result should be read with the caveat that nearly all counties were represented in the sample. The value of Causal Mplus Trees in analyzing these data is evident in its ability to find a group of counties exhibiting stronger party effects, while simultaneously matching on COVID-19 trajectories.

Our findings corroborate those of previous COVID-19 partisanship studies. Allcott et al. (2020) found evidence of 3.6 percent fewer point of interest visits associated with a 10 percentage point decrease in the Republican vote share (roughly equivalent to shifting from the median to the 25th percentile Republican vote share county for the 2010 presidential election). Brzezinski et al. (2020) estimated a 3 percentage point difference in the share of devices staying fully at home for the 90th vs 10 th percentile Democrat vote share counties 15 days after a county's first case. Areas with relatively greater viewership of conservative news shows that initially downplayed the threat of coronavirus (versus those that accurately portrayed the pandemic) have also been linked to delayed behavior changes and higher initial occurrences of cases and deaths (Bursztyn, Rao, Roth, \& Yanagizawa-Drott, 2020). Further, our Group 2 CATE is comparable in magnitude to the decline in travel distance attributable to statewide stayat-home mandates (Sears et al., 2020). While prior studies employ traditional approaches for discussing treatment effect heterogeneity (i.e. running differencein-differences or event study regressions on subgroups of interest), the Causal $\mathrm{M}$ plus Trees method provides a data-driven approach to identifying comparable groups on model fit and analyzing treatment effect heterogeneity.

\section{Discussion}

In this paper, we proposed the Causal Mplus Trees algorithm, which matches on parameter estimates of an SEM using a tree-based approach and uses these groupings to estimate CATEs in a holdout sample. We used two small simulation studies to demonstrate a proof of concept for the approach. We also showed how it could be used to estimate party effects on mobility using COVID-19 data. We reiterate that we do not see Causal Mplus Trees as a substitute for traditional matching methods. Propensity score matching and related methods have their place and can be effective in matching on covariates, both observed and latent. We believe that our approach offers an alternative option to those whose research questions would be better addressed by the ability to match on parameter estimates from an SEM.

\subsection{Practical Recommendations}

We encourage users of Causal Mplus Trees to carefully consider how they select and differentiate between modeled covariates and splitting covariates. Although 
the procedure ultimately matches on both, the way it does so differs by covariate type. Matching is performed on modeled covariates indirectly through the parameter estimates produced by the model, whereas splitting covariates are matched more directly on the observed values of the scores. The choice of whether a covariate should be used as a modeled or splitting covariate depends upon what specifically the user wants to match, which can vary based on the research question, study design, and characteristics of the sample collected.

Another consideration for researchers using Causal Mplus Trees is the depth to which the tree should be grown. Cross-validation is the most commonly used approach for this in the context of conventional decision trees. However, we believe that cross-validation may not be as well suited for our purposes primarily because it is designed to optimize predictive accuracy. In our algorithm, the goal of the tree is not to optimize predictive accuracy, but rather to partition the sample into groups that are matched well enough on $\hat{\boldsymbol{\theta}}$ to justify causal inference in the holdout sample. As in propensity score matching, there is no objective criterion for this, so the researcher must make a subjective judgment and make a case to justify it.

We urge researchers to take into account the following considerations. First, the sample size in each parent node must be large enough to estimate $M$ in not only the parent node, but also each of the daughter nodes. SEMs can require larger sample sizes to estimate, so limits should be placed on the splitting procedure so as not to consider splitting on a sample that does not have a large enough sample to do this. Related to this is the need for a sufficient number of treated and untreated participants in each terminal node to be able to estimate the CATEs in the holdout sample. If a group has no treated (or no untreated) participants, the CATE cannot be estimated. Of course, it is possible that the mix in the tree differs from the mix in the holdout sample, but to the extent that the matching subsample is a reflection of the estimation subsample, the matching subsample can give a sense of the mix one would expect in the estimation subsample. If performing hypothesis tests, certain minimum sample sizes are required to meet the assumptions of the test as well as to detect the effects, so these must also be kept in mind when deciding how deep to grow the tree.

Parsimony is also important to consider, especially with respect to building a coherent narrative with policy implications. We are typically searching for groups with qualitative meaning given the relevant theoretical framework. If the tree were to produce a dozen groups, it may be challenging to map this onto available theory in order to interpret the results. The relative importance of parameters in characterizing a pattern should be taken into account as well. Theory may dictate that some parameters may be more important to match on than others for a given context (e.g., the residual variance in our stability example). As such, it could be justifiable to trim the tree earlier if splits begin resulting in differences in less relevant parameters. The size of parameter estimates may also play a role. For example, the algorithm could decide on a split that results in two daughter nodes with only small differences in their parameter estimates. Treating these as two separate groups for the purpose of estimating the CATE may not be 
worthwhile. Similar to the logic used in propensity score analysis, the treated and untreated participants in each node should be compared on their parameters estimates, to verify, even if only subjectively, that they are similar and therefore matched to some degree.

The choice for the depth of the tree depends on a trade-off between interpretability of a result and the validity of the causal inference. If one were to view the ability to draw causal inference as how well treated and untreated participants are matched, then the ability to draw causal inference can be conceptualized not as a dichotomy but as a continuum with perfectly matched participants on one end and perfectly unmatched participants on the other. The better matched participants are, the greater the ability to draw causal inference. However, better matching requires a deeper tree, which becomes less interpretable and generalizable as the depth grows. This trade-off exists in propensity score matching as well but is more apparent in the context of decision trees where such trade-offs are more apparent and a language with which to conceptualize and discuss them already exists.

\subsection{Future Research and Conclusions}

Plenty of opportunities exist to expand on this work. Although two simulation studies were conducted, they only served as a proof of concept. Additional simulations would be helpful in evaluating the effectiveness of the algorithm across a variety of conditions. The causal tree approach has been extended to use random forests (Wager \& Athey, 2018), which are known to be more stable than decision trees. These causal forests have also been modified to accommodate multilevel data structures (Suk, Kang, \& Kim, in press). SEM Trees have been expanded to SEM Forests (Brandmaier, Prindle, McArdle, \& Lindenberger, 2016), so expanding our algorithm to use random forests would be a natural next step. Additionally, we note that our discussion of treatment effects was limited to mean differences in univariate outcomes. However, given that SEM is already being employed as well as the flexibility of Causal Mplus Trees, it is possible that the outcome measure could be generalized to the multivariate context, with treated and untreated participants being compared on a model using, for example, a multiple group SEM. To conclude, we believe our proposed algorithm can provide researchers with the opportunity to match on SEM parameter estimates, thereby allowing them greater flexibility in what they can match on and the kinds of research questions they can address as a result.

\section{References}

Abrevaya, J., Hsu, Y.-C., \& Lieli, R. (2015). Estimating conditional average treatment effects. Journal of Business and Economic Statistics, 33, 485-505. doi: https://doi.org/10.1080/07350015.2014.975555

Adolph, C., Amano, K., Bang-Jensen, B., Fullman, N., \& Wilkerson, J. (2020). Pandemic politics: Timing state-level social distancing responses to COVID-19. medRxiv. doi: https://doi.org/10.1101/2020.03.30.20046326 
Allcott, H., Boxell, L., Conway, J., Gentzkow, M., Thaler, M., \& Yang, D. (2020). Polarization and public health: Partisan differences in social distancing during the coronavirus pandemic. Journal of Public Economics, 191. doi: https://doi.org/10.1016/j.jpubeco.2020.104254

Athey, S., \& Imbens, G. (2016). Recursive partitioning for heterogeneous causal effects. Proceedings of the National Academy of Sciences, 113, 7353-7360. doi: https://doi.org/10.1073/pnas.1510489113

Austin, P. (2009). The relative ability of different propensity-score methods to balance measured covariates between treated and untreated subjects in observational studies. Medical Decision Making, 29, 661-677. doi: https://doi.org/10.1177/0272989X09341755

Austin, P. (2011). An introduction to propensity score methods for reducing the effects of confounding in observational studies. Multivariate Behavioral Research , 46, 399-424. doi: https://doi.org/10.1080/00273171.2011.568786

Berk, R. (2004). Regression analysis: A constructive critique. Sage. doi: https://doi.org/10.4135/9781483348834

Brandmaier, A., Oertzen, T., McArdle, J., \& Lindenberger, U. (2013). Structural equation model trees. Psychological Methods, 18, 71-86. doi: https://doi.org/10.1037/a0030001.

Brandmaier, A., Prindle, J., \& Arnold, M. (2021). semtree: Recursive partitioning for structural equation models [ $\mathrm{R}$ package version 0.9.17.]. Retrieved from https://CRAN.R-project.org/package=semtree

Brandmaier, A., Prindle, J., McArdle, J., \& Lindenberger, U. (2016). Theoryguided exploration with structural equation model forests. Psychological Methods, 21, 566-582. doi: https://doi.org/10.1037/met0000090

Breiman, L., Friedman, J., Olshen, R., \& Stone, C. (1984). Classification and regression trees. Chapman \& Hall. doi: https://doi.org/10.1201/9781315139470

Browne, M., \& Toit, S. (1991). Models for learning data. In L. Collins \& J. Horn (Eds.), Best methods for the analysis of change (p. 47-68). American Psychological Association. doi: https://doi.org/10.1037/10099-004

Brzezinski, A., Deiana, G., Kecht, V., \& Van Dijcke, D. (2020). The covid-19 pandemic: Government vs. community action across the united states. INET Oxford Working Paper. Retrieved from https://www.inet.ox.ac.uk/ publications/no-2020-06-the-covid-19-pandemic-government-vs -community-action-across-the-united-states/ (No. 2020-06.)

Bursztyn, L., Rao, A., Roth, C., \& Yanagizawa-Drott, D. (2020). Misinformation during a pandemic. NBER Working Paper(27417). doi: https://doi.org/10.3386/w27417

Dong, E., Du, H., \& Gardner, L. (2020). An interactive web-based dashboard to track covid-19 in real time. Lancet Infectious Disease, 20, 533-534. doi: https://doi.org/10.1016/S1473-3099(20)30120-1

Ferrer, E. (2016). Exploratory approaches for studying social interactions, dynamics, and multivariate processes in psychological science. Multivariate Behavioral Research, 51, 240-256. doi: 
https://doi.org/10.1080/00273171.2016.1140629

Ferrer, E., Steele, J., \& Hsieh, F. (2012). Analyzing dynamics of affective dyadic interactions using patterns of intra- and inter-individual variability. Multivariate Behavioral Research, 47, 136-171. doi: https://doi.org/10.1080/00273171.2012.640605

Gadarian, S., Goodman, S., \& Pepinsky, T. (2020). Partisanship, health behavior, and policy attitudes in the early stages of the COVID-19 pandemic. SSRN. doi: https://doi.org/10.2139/ssrn.3562796

Greenland, S., Pearl, J., \& Robins, J. (1999). Causal diagrams for epidemiologic research. Epidemiology, 10, 37-48. doi: https://doi.org/10.1097/00001648199901000-00008

Grimm, K., \& Ram, N. (2009). Nonlinear growth models in mplus and sas. Structural Equation Modeling, 16, 676-701. doi: https://doi.org/10.1080/10705510903206055

$\mathrm{Gu}, \quad$ X., \& Rosenbaum, P. (1993). Comparison of multivariate matching methods: Structures, distances, and algorithms. Journal of Computational and Graphical Statistics, 2, 405-420. doi: https://doi.org/10.1080/10618600.1993.10474623

Guo, S., \& Fraser, M. (2010). Propensity score analysis: Statistical methods and applications. Sage.

Hallquist, M., \& Wiley, J. (2018). MplusAutomation: An R package for facilitating large-scale latent variable analyses in Mplus. Structural Equation Modeling, 25, 621-638. doi: https://doi.org/10.1080/10705511.2017.1402334

Harder, V., Stuart, E., \& Anthony, J. (2010). Propensity score techniques and the assessment of measured covariate balance to test causal associations in psychological research. Psychological Methods, 15, 234-249. doi: https://doi.org/10.1037/a0019623

Hirano, K., \& Imbens, G. (2001). Estimation of causal effects using propensity score weighting: An application to data on right heart catheterization. Health Services and Outcomes Research Methodology, 2, 259-278. doi: https://doi.org/10.1023/A:1020371312283

Ho, D., Imai, K., King, G., \& Stuart, E. (2007). Matching as nonparametric preprocessing for reducing model dependence in parametric causal inference. Political Analysis, 15, 199-236. doi: https://doi.org/10.1093/pan/mpl013

Holland, P. (1986). Statistics and causal inference. Journal of the American Statistical Association, 81, 945-60. doi: https://doi.org/10.1080/01621459.1986.10478354

Jöreskog, K. (1971). Simultaneous factor analysis in several populations. Psychometrika, 36, 409-426. doi: https://doi.org/10.1007/BF02291366

Lee, B., Lessler, J., \& Stuart, E. (2010). Improving propensity score weighting using machine learning. Statistics in Medicine, 29, 337-346. doi: https://doi.org/10.1002/sim.3782

Leite, W., Stapleton, L., \& Bettini, E. (2018). Propensity score analysis of complex survey data with structural equation modeling: A tutorial with mplus. Structural Equation Modeling, 3, 448-469. doi: 
https://doi.org/10.1080/10705511.2018.1522591

Meredith, W., \& Tisak, J. (1990). Latent curve analysis. Psychometrika, 55, 107-122. doi: https://doi.org/10.1007/bf02294746

Muthén, L., \& Muthén, B. (1998-2017). Mplus user's guide (8th ed.) [Computer software manual]. Muthén \& Muthén.

National Governors Association. (2020). Governors roster. Retrieved from https://www.nga.org/wp-content/uploads/2019/07/Governors -Roster.pdf

Neale, M., Hunter, M., Pritikin, J., Zahery, M., Brick, T., Kirkpatrick, R., \& Boker, S. (2016). Openmx 2.0: Extended structural equation and statistical modeling. Psychometrika, 81, 535-549. doi: https://doi.org/10.1007/s11336-014-9435-8

Neyman, J., Iwaszkiewicz, K., \& Kolodziejczyk, S. (1935). Statistical problems in agricultural experimentation. Supplement to the Journal of the Royal Statistical Society, 2, 107-180. doi: https://doi.org/10.2307/2983637

Normand, S., Landrum, M., Guadagnoli, E., Ayanian, J., Ryan, T., Cleary, P., \& McNeil, B. (2001). Validating recommendations for coronary angiography following an acute myocardial infarction in the elderly: A matched analysis using propensity scores. Journal of Clinical Epidemiology, 54, 387-398. doi: https://doi.org/10.1016/S0895-4356(00)00321-8

R Core Team. (2020). R: A language and environment for statistical computing [Computer software manual]. Vienna, Austria. Retrieved from https:// www.R-project.org/

Raykov, T. (2012). Propensity score analysis with fallible covariates: A note on a latent variable modeling approach. Educational and Psychological Measurement, 72, 715-733. doi: https://doi.org/10.1177/0013164412440999

Rosenbaum, P., \& Rubin, D. (1983). The central role of the propensity score in observational studies for causal effects. Biometrika, 70, 41-55. doi: https://doi.org/10.1093/biomet/70.1.41

Rosenbaum, P., \& Rubin, D. (1984). Reducing bias in observational studies using subclassification on the propensity score. Journal of the American Statistical Association, 79, 516-24. doi: https://doi.org/10.2307/2288398

Rosenbaum, P., \& Rubin, D. (1985). The bias due to incomplete matching. Biometrics, 41, 103-16. doi: https://doi.org/10.2307/2530647

Rosseel, Y. (2012). lavaan: An R package for structural equation modeling. Journal of Statistical Software, 48(2), 1-36. doi: https://doi.org/10.18637/jss.v048.i02

Rubin, D. (1974). Estimating causal effects of treatments in randomized and nonrandomized studies. Journal of Educational Psychology, 66, 688-701. doi: https://doi.org/10.1037/h0037350

Rubin, D. (1980). Randomization analysis of experimental data: The fisher randomization test comment. Journal of the American Statistical Association, 75, 591-593. doi: https://doi.org/10.2307/2287653

Rubin, D. (1986). What if's have causal answers. Journal of the American Statistical Association, 81, 961-962. doi: 
https://doi.org/10.1080/01621459.1986.10478355

Sears, J., Villas-Boas, S., Villas-Boas, M., \& Villas-Boas, V. (2020). Are we \#stayinghome to flatten the curve? SSRN. doi: https://doi.org/10.2139/ssrn.3569791

Serang, S., Jacobucci, R., Stegmann, G., Brandmaier, A., Culianos, D., \& Grimm, K. (2021). Mplus Trees: Structural equation model trees using Mplus. Structural Equation Modeling, 28, 127-137. doi: https://doi.org/10.1080/10705511.2020.1726179

Stegmann, G., Jacobucci, R., Serang, S., \& Grimm, K. (2018). Recursive partitioning with nonlinear models of change. Multivariate Behavioral Research, 53, 559-570. doi: https://doi.org/10.1080/00273171.2018.1461602

Suk, Y., Kang, H., \& Kim, J.-S. (in press). Random forests approach for causal inference with clustered observational data. Multivariate Behavioral Research. doi: https://doi.org/10.1080/00273171.2020.1808437

Therneau, T., \& Atkinson, B. (2018). Rpart: Recursive partitioning and regression trees [ $\mathrm{R}$ package version 4.1-13.]. Retrieved from https:// CRAN . R-project . org $/$ package $=$ rpart

Thoemmes, F., \& Kim, E. (2011). A systematic review of propensity score methods in the social sciences. Multivariate Behavioral Research, 46, 90-118. doi: https://doi.org/10.1080/00273171.2011.540475

Unacast. (2020). Unacast social distancing scoreboard dataset. Retrieved from https://www . unacast. com/data-for-good.

U.S. Census Bureau. (2010). Decennial census, 2010. Retrieved from https:// data. census.gov/

U.S. Department of Education, Institute of Education Sciences, \& What Works Clearinghouse. (2017). What works clearinghouse: Standards handbook (version 4.0). Retrieved from https://ies.ed.gov/ncee/wwc/Docs/ referenceresources/wwc_standards_\handbook_v4.pdf

Wager, S., \& Athey, S. (2018). Estimation and inference of heterogeneous treatment effects using random forests. Journal of the American Statistical Association, 113, 1228-1242. doi: https://doi.org/10.1080/01621459.2017.1319839

West, S., Cham, H., Thoemmes, F., Renneberg, B., Schulze, J., \& Weiler, M. (2014). Propensity scores as a basis for equating groups: Basic principles and application in clinical treatment outcome research. Journal of Consulting and Clinical Psychology, 82, 906-919. doi: https://doi.org/10.1037/a0036387 